\title{
IMPROVED PERFormanCE BY PARAMETRIZING WAVELET FILTERS FOR DIGITAL IMAGE WATERMARKING
}

\author{
MANGAIYARKARASI PALANIVEL ${ }^{1}$ AND ARULSELVI SHANMUGAM ${ }^{2}$ \\ 1 and 2 Department of Electronics \& Instrumentation Engineering, Annamalai University, \\ Annamalai Nagar, Chidambaram, India \\ mangai_me@yahoo.co.in
}

\begin{abstract}
In recent years, watermarking has become an attractive field in areas like copyright protection, image authentication and biomedical engineering. Many literatures have reported about discrete wavelet transform (DWT) watermarking techniques for data security. However, DWT based watermarking schemes are found to be less robust against image processing attacks. In this paper, an attempt is made to develop a scheme based on Redundant Discrete Wavelet Transform (RDWT). The robustness and security of the proposed RDWT scheme is improved by parametrizing the scaling and wavelet filters by Pollen's parametrizing technique. An Independent Component Analysis (ICA) based detector is also applied, which extracts watermark directly in spatial domain rather than in transform domain. The unique feature of ICA is that it does not require any transformation during extraction. The results reveal that the proposed scheme produces high peak signal to noise ratio (PSNR) values and similarity measure under various image processing attacks.
\end{abstract}

\section{KEYWORDS}

Digital Watermarking, Filter Parametrization, Independent Component Analysis (ICA) and Redundant Discrete Wavelet Transform (RDWT).

\section{INTRODUCTION}

With the rapid growth of the internet and the development of digital multimedia technologies, exchange and copy of digital multimedia have become quite convenient [1]. Hence, the copyright protection of digital multimedia has become an important issue. A technique to solve this problem is digital watermarking, which embeds directly some digitized information into digital media by making small modification to the media, where the watermark information remains detectable after attack. Thus the digital watermarking can be used to identify the rightful owner [2]. Furthermore, it is an important issue to develop a robust watermarking scheme with a better trade-off between robustness and imperceptibility. Recently many literatures have reported the watermarking schemes based on DWT [3]. Among these schemes, the one which require the original information and secret keys for the watermarking extraction are called private watermark schemes. Schemes which require the watermark information and secret keys are called semiprivate or semi-blind schemes. Schemes which need secret keys rather than the original information are called public or blind watermark schemes.

In general, the robustness of private watermark scheme is good to endure signal processing attacks. However, they are not feasible in real application, such as DVD copy protection where the original information may not be available for watermark detection. On the other hand, semiblind and blind watermark schemes are more feasible in that situation. However, they have lower robustness than the private watermark schemes. 
Signal \& Image Processing : An International Journal (SIPIJ) Vol.3, No.1, February 2012

Watermarking techniques can be divided into two main groups: i) embedding watermarks in the spatial domain and ii) frequency domain. Spatial domain watermarking directly embeds the watermark into the object while frequency domain embeds the watermark by changing frequency component values by an orthogonal transformation. DWT based watermarking is a widely used technique to embed a spread spectrum watermark into DWT coefficients. Moreover, the conventional watermark detection uses transform techniques to decompose the corrupted image, of which the ownership is determined, and from which the watermark is recovered. In general, toward the aim of watermark recovery, some detection systems require previous knowledge of the watermark such as its location, the strength, the threshold or the original image. Also in some cases, the watermarking system requires embedding multi- information such as sources, authors, creators, owners, distributors or authorized consumers of a document. Therefore, a watermarking algorithm must be able to embed watermarks to satisfy the above requirements [4].

A new approach is proposed in this paper, where the watermarking method is based on the use of filter parametrized RDWT for hiding the information. In general, RDWT produce an over complete, over sampled expansion system which is used to embed watermark to the transformed coefficients. The filters used in the transformation process are designed using Pollen's filter parametrizing technique. The perceptual model is applied with stochastic approach for watermark embedding. This is based on computation of a noise visibility function (NVF) which has local image properties where the strength of watermarking is controlled. The result is that watermarks at texture and edge areas are stronger than flat areas. Besides, an intelligent detection technique based on ICA is implemented for extraction without the use of previous knowledge of the watermark and even the transformation process [5]. Robustness against various attacks like filtering, cropping, JPEG compression and transparencies of the proposed scheme are demonstrated with simulation results.

The paper is organized as follows: Section 2 reviews the RDWT and its filter parametrization. Section 3I discusses the watermarking embedding algorithm. In section 4 , watermark extraction technique using ICA is explained. Simulation results are presented in section 5 and conclusions are drawn in section 6.

\section{REDUNDANT DisCRETE WAVELET TRANSFORM}

This section briefly reviews the image decomposition methods based on discrete wavelet transform.

\subsection{Two dimensional decomposition methods}

A two dimensional DWT is shown in Fig. 1(a), where $h[-m]$ and $g[-m]$ are the low pass and high pass analysis filters, while the corresponding low pass and high pass synthesis filters are $h[m]$ and $g[m] ; c_{j}$ and $d_{j}$ are the low band and high band output coefficients at level $\mathbf{j}$.

DWT analysis is given by

$$
\begin{gathered}
c_{j}[m, n]=\left\langle c_{j+1}(m, n) * h[-m]\right) \downarrow 2 \\
d_{j}[m, n]=\left\langle c_{j+1}(m, n) * g[-m]\right) \downarrow 2
\end{gathered}
$$

Similarly DWT synthesis is given by

$$
c_{j+1}[m, n]=\left[\left(c_{j}(m, n) \uparrow 2\right) * h[m]+\left(d_{j}(m, n) \uparrow 2\right) * g[m]\right]
$$


where $*$ denotes convolution, $\downarrow 2$ and $\uparrow 2$ denotes downsampling and upsampling by a factor of two.

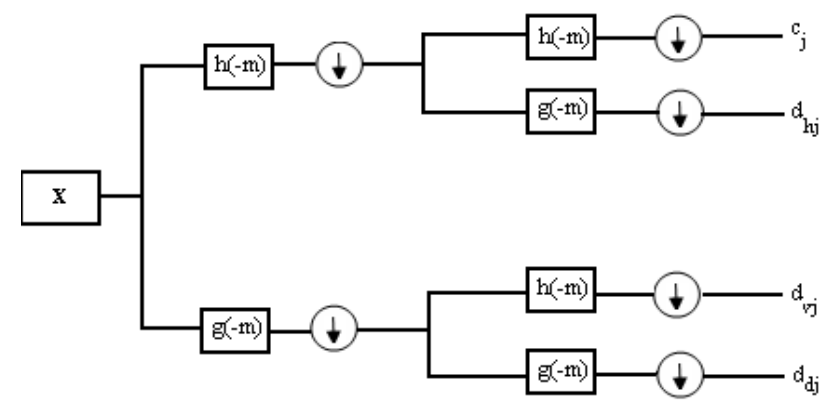

Fig. 1(a). A two dimensional decomposition using DWT.

However, the shift variant characteristic of DWT implies the lack of samples during reconstruction. Unlike DWT, RDWT gives an over complete representation of the input sequence and functions as a better approximation to the continuous wavelet transform. The RDWT is shift invariant, and its redundancy introduces an over complete frame expansion. It is known that frame expansion increases the robustness to additive noise, that is, addition of noise to transform coefficient results in less signal distortion for frame expansions than for orthogonal expansion [6]. RDWT has been proposed for signal detection and enhancement, since the RDWT maintains uniform sampling rate in time domain and in some respects, is a discrete approximation to the continuous wavelet transform.

RDWT removes the down-sampling operation from the traditional critically sampled DWT. To describe the implementation of the RDWT in terms of DWT is illustrated below:

In contrast, a two dimensional RDWT is shown in Fig. 1(b). The RDWT eliminates downsampling and upsampling of coefficients, and at each scale, the number of output coefficients doubles that of the input. The filters themselves are up-sampled to fit the growing data length. Specifically, the filters for scale $j$ are

$$
\begin{aligned}
h_{j}[m] & =h_{j+1}[m] \uparrow 2 \\
g_{j}[m] & =g_{j+1}[m] \uparrow 2
\end{aligned}
$$

The RDWT multi-resolution analysis is given by

$$
\begin{array}{r}
c_{j}[m, n]=\left(c_{j+1}(m, n) * h[-m]\right) \\
d_{j}[m, n]=\left(c_{j+1}(m, n) * g[-m]\right)
\end{array}
$$

while the RDWT synthesis is given by

$$
c_{j+1}[m, n]=\frac{1}{2}\left[\left(c_{j}(m, n)\right) * h[m]+\left(d_{j}(m, n)\right) * g[m]\right]
$$

Lack of down-sampling in the RDWT analysis yields a redundant representation of the input sequence. Specifically, two valid descriptions of the coefficients exist after one stage of RDWT analysis. The direct implementation of RDWT given by (6), (7) and (8) results in sub-bands that are exactly the same size as the original image as shown in Fig. 2. The advantage of this representation is that each RDWT coefficient is located within its sub-band in its spatially correct position. By appropriately sub-sampling each sub-band of an RDWT, one can produce exactly the 
same coefficients as does a critically sampled DWT applied to the same input signal. In Fig. 1(b), a $j$-scale two dimensional RDWT consists of $4 j$ distinct critically sampled DWTs.

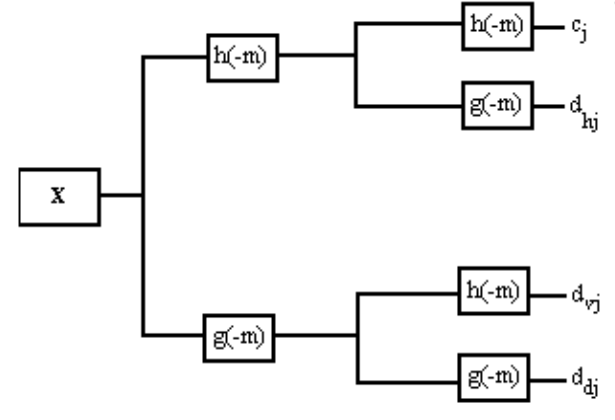

Fig. 1(b). A two dimensional decomposition using RDWT.

Fig. 2 shows spatially coherent representation of a two scale two dimensional RDWT, where coefficients retain their correct spatial location within each sub-band image is the same size as the original image such as $L L_{j}, H L_{j}, L H_{j}$ and $H H_{\mathrm{j}}$ denote the approximation, horizontal, vertical and diagonal sub-bands, respectively at scale $j$.

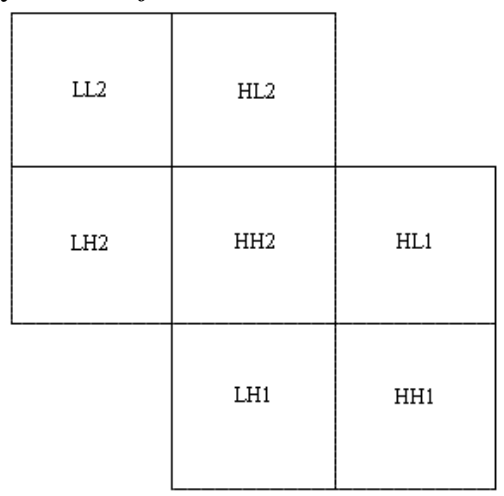

Fig. 2. Spatially coherent representation of a two scale two dimensional RDWT.

\subsection{Filter Parametrization}

In this paper, the host image is decomposed using wavelet filters constructed with Pollen's parametrization technique given below [7]. The construction of a 6-tap orthogonal filter is given by

$$
\begin{aligned}
a_{-2}= & ((1+\cos \alpha+\sin \alpha) *(1-\cos \beta-\sin \beta) \\
& +2 * \sin \beta \cos \alpha) / 4 \\
a_{-1}= & ((1-\cos \alpha+\sin \alpha) *(1+\cos \beta-\sin \beta) \\
& -2 * \sin \beta \cos \alpha) / 4 \\
a_{0}= & (1+\cos (\alpha-\beta)+\sin (\alpha-\beta)) / 2 \\
a_{1}= & (1+\cos (\alpha-\beta)-\sin (\alpha-\beta)) / 2 \\
a_{2}= & 1-a_{-2}-a_{0} \\
a_{3}= & 1-a_{-1}-a_{1}
\end{aligned}
$$

where $\alpha$ and $\beta$ lies between $-\pi \leq \alpha, \beta<\pi$. 
The parameter values $\alpha$ and $\beta$ used for construction of filters and the resulting wavelet filter coefficients are kept secret. Hence, the watermark information can be embedded in a secret multiresolution transform domain, making it difficult to mount a hostile attack that seeks to destroy or remove watermark information at specific locations.

\section{WATERMARK EMBEDDING}

The original image is decomposed into two levels using RDWT as shown in Fig.1 (b). The approximation components at $L L_{2}$ sub-band are not chosen to embed watermark because they will seriously degrade image quality. Similarly the diagonal detail coefficients $\mathrm{HH}_{2}$ are also not considered because security is poor when watermark is embedded.

Hence, the middle subbands $H L_{2}$ and $L H_{2}$ are chosen in this work, to embed watermark based on the trade off between imperceptibility and robustness. A stochastic model of the cover image is applied to an adaptive watermark by computing NVF with non-stationary Gaussian model [8]. In this case, NVF can be expressed by

$$
\mathrm{NVF}(\mathrm{i}, \mathrm{j})=\frac{1}{1+\sigma_{x}^{2}(i, j)}
$$

where $\sigma_{x}^{2}(i, j)$ denotes variance of the cover image in a window centered on the pixel with coordinates $(i, j)$. By applying NVF, the watermark in texture and edges becomes stronger than in flat areas. The watermark is embedded using the following equations:

$$
\begin{aligned}
& I^{\prime} L H_{2}(i, j)=L H_{2}(i, j)+E\left(L H_{2}\right) \alpha_{1}(1-N V F(i, j)) W(i, j)+\frac{E\left(L H_{2}\right)}{10} \cdot \alpha_{2} \cdot N V F(i, j) \cdot W(i, j) \\
& I^{\prime} H L_{2}(i, j)=H L_{2}(i, j)+E\left(H L_{2}\right) \alpha_{1}(1-N V F(i, j)) W(i, j)+\frac{E\left(H L_{2}\right)}{10} \cdot \alpha_{2} \cdot N V F(i, j) \cdot W(i, j)
\end{aligned}
$$

where $I^{\prime} L H_{2}(i, j)$ and $I^{\prime} H L_{2}(i, j)$ are watermarked transform coefficients, $E\left(L H_{2}\right) \alpha_{1}$ and $E\left(H L_{2}\right) \alpha_{1}$ denote the watermark strengths of texture and $\frac{E\left(L H_{2}\right)}{10} \cdot \alpha_{2}$ and $\frac{E\left(H L_{2}\right)}{10} \cdot \alpha_{2}$ denote the watermark strengths of edge regions for $L H$ and $H L$ subbands, respectively. $\alpha_{1}$ and $\alpha_{2}$ are smoothing factors at the texture regions and flat regions and $E$ denotes the mean and $W(i, j)$ is the watermark.To retrieve the watermarked image, inverse RDWT is performed. In order to apply ICA for watermark detection, the embedding process needs to create a private key for extraction.

\section{WATERMARK EXTRACTION}

This section briefly reviews ICA algorithm and how ICA is applied to watermark extraction [9]. ICA aims at extracting unknown hidden components from multivariate data using only the assumption that the unknown factors are mutually independent. The general ICA model can be described as follows:

The ICA algorithm starts with the assumption that data vector $X=\left(X_{1}, \ldots, X_{M}\right)$ can be represented in terms of linear superposition of basis functions, as given by

$$
\begin{aligned}
& X=A . S \\
& Y=B . X
\end{aligned}
$$


Signal \& Image Processing : An International Journal (SIPIJ) Vol.3, No.1, February 2012

where $S=\left(S_{1}, \ldots, S_{N}\right)$ are unknown source coefficients, A is an $M X N$ matrix and the columns $\mathrm{a}_{1}, \ldots, \mathrm{a}_{\mathrm{N}}$ are called basis functions. Basis functions are constant while coefficients vary with data. The goal of ICA is to find matrix $B$ in (14), which results in estimates of coefficient values of $Y$ being statistically as independent as possible over a set of data $X$.

In this paper, FastICA algorithm is used for watermark extraction [8]. It is noted that the watermark and the original image can be regarded as unknown sources $\left(S_{i}\right)$ and the watermark as a mixture $\left(X_{i}\right)$. By creating different mixtures, one can perform ICA to extract the watermark.

The data is first processed for centering and whitening, thereby the observer variable $X$ is centered by subtracting the mean vector $m=E\{X\}$. The above mentioned processing is designed to simplify the ICA algorithms. After estimating the mixing matrix A with centered data, we can complete the estimation by adding the mean vector of the original source signal back to the centered estimation of the source data. The FastICA algorithm is applied to the image that has been centered to find the separate matrix $B$. The features of FastICA are fast convergence, easy to implement and suitable for watermark applications.

To perform ICA, a linear mix of image with keys is generated to demix the watermark signal from the mixtures. The novelty of this detector is that it does not require the transform process to separate $L H$ and $H L$ bands for watermark extraction and omits using original and embedding parameters such as watermark location and strength. In this paper, watermarks are embedded in RDWT sub-band and the location of embedding is the same in spatial domain. Therefore ICA is applied directly on the watermarked image. With the help of a private key $K_{p}$ to create different mixtures, one can extract successfully the watermark to claim the ownership.

Mixtures are created by the following equations:

$$
\begin{aligned}
& X_{1}(i, j)=I^{\prime}(i, j)+K_{p}(i, j) \\
& X_{2}(i, j)=K(i, j)+K_{p}(i, j) \\
& X_{3}(i, j)=I^{\prime}(i, j)
\end{aligned}
$$

where $X_{1}, X_{2}, X_{3}$ are mixtures, $I^{\prime}$ is the watermarked image and $K_{p}$ is a key in watermark embedding process. Those mixtures can be modeled as

$$
\begin{aligned}
& X_{1}=a_{11} I+a_{12} W+a_{13} K \\
& X_{2}=a_{21} I+a_{22} W+a_{23} K \\
& X_{3}=a_{31} I+a_{32} W+a_{33} K
\end{aligned}
$$

where $a$ is a mixing matrix, $W$ is the watermark matrix and $K$ is a random key in the embedding process. Applying the FastICA algorithm to those mixtures, matrix watermark $W$ is extracted. This paper discusses the FastICA algorithm to extract the watermark.

\section{Simulation RESUlts}

The proposed watermarking is tested on $256 \times 256$ flower image. Daubechies 3 filter is compared with the parametrized filter for two levels. Highest middle frequency sub-bands $\mathrm{ILH}_{2}(i, j)$ and $\mathrm{IHL}_{2}(i, j)$ are selected to embed watermark. A watermark of same size of original 
image is used and $\alpha_{1}, \alpha_{2}$ were set to 0.1 by repeated simulation to ensure the invisibility of the watermark. Fig. 4 shows the original image and Fig. 5 shows the watermark. Fig. 6(a) and (b) are watermarked image using db3 filter and parametrized filter respectively. Both original and watermarked images are evidently indistinguishable and sharper in Fig. 6(b) when compared to Fig. 6(a). This shows that imperceptibility is improved better in parametrized filter when compared to the db3 filter. The images have PSNR values of $51.87 \mathrm{~dB}$ and $56.45 \mathrm{~dB}$ for $\mathrm{db} 3$ and parametrized filters respectively.

Similarly, the watermark detection using ICA extracts the watermark perfectly from the watermarked image. Fig. 7 (a) and (b) shows the extracted watermark from the watermarked images obtained by db3 filter and parametrized filter respectively. The result confirms that the ICA detector can extract the watermark from both the images perfectly. The PSNR values of watermarked image should vary from image to image because of the effectiveness of NVF, $\alpha_{1}$ and $\alpha_{2}$. They are used to control the invisibility.

The robustness of the proposed watermarking scheme is validated against attacks like Salt \& Pepper noise, Gaussian noise additions and Cropping. Fig. 8 (a) shows the watermarked image with addition of Salt $\&$ Pepper noise with noise density of 0.05 and Fig. 8 (b) shows the addition of Gaussian noise with mean value 0 and variance 0.05 . Fig. 8 (c ) shows the median filtered image of window size $5 \times 5$. The watermarked image with cropping, where the pixels of the watermarked image are replaced by pixels of another image is shown in Fig. 8(d). From the extracted results obtained after the above mentioned attacks, it is inferred that the RDWT using parametrized filter possess considerable improvement in PSNR when compared to the existing daubechies filters. The comparison made in Table 1 shows the robustness of the proposed scheme against various attacks. Table 2 shows the ability of the ICA detector that perfectly extracts the watermark from watermarked image in the presence of attacks.

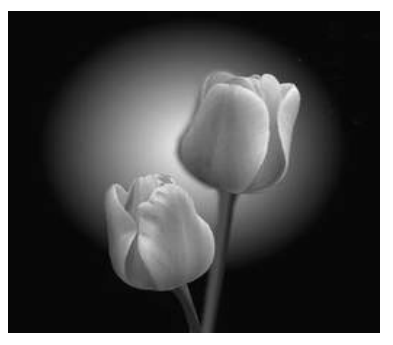

Fig. 4. Original image.

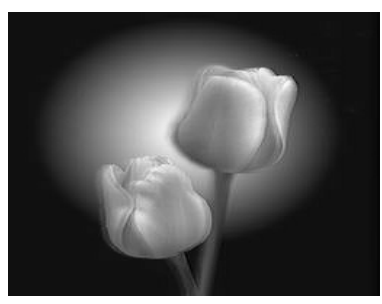

(a)

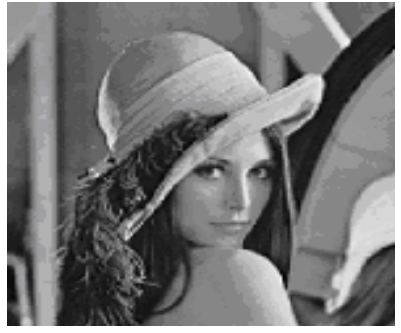

Fig. 5. Watermark.

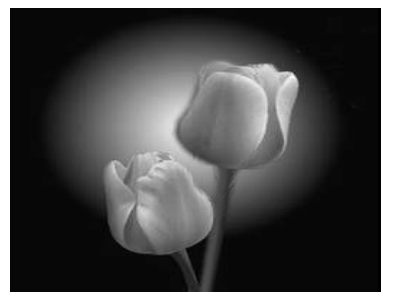

(b)

Fig. 6. Watermarked image using (a) dB3 filter and (b) parametrized filter 
Signal \& Image Processing : An International Journal (SIPIJ) Vol.3, No.1, February 2012
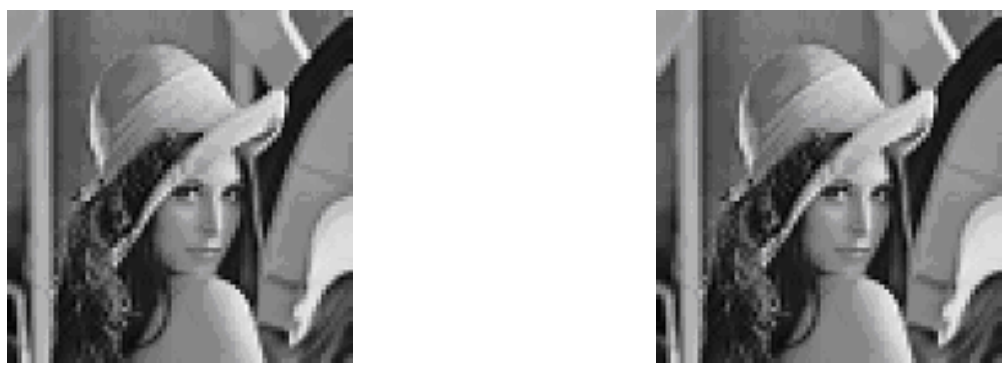

Fig. 7. Extracted watermark from Fig. 6 (a) and (b).

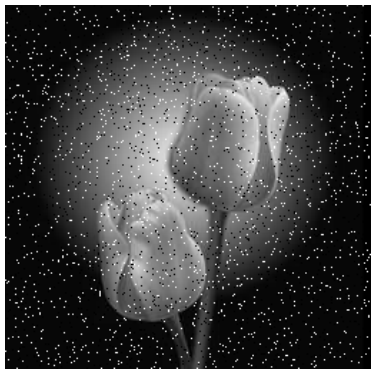

(a) Salt \& Pepper noise added (mean=0,var=0.05)

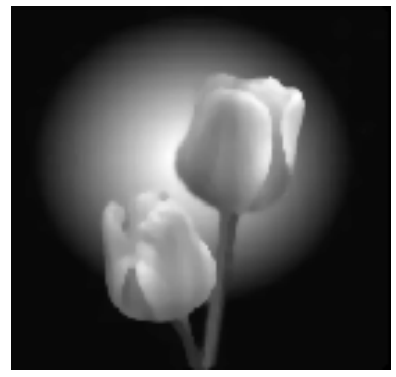

(c) Median filtered (5x5).

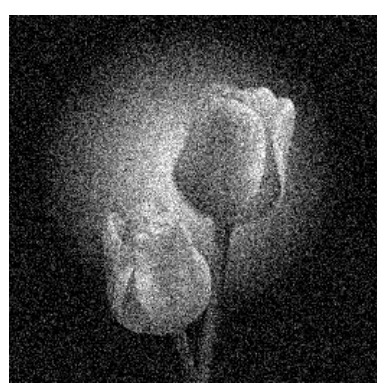

(b) Gaussian noise added ( 0.05).

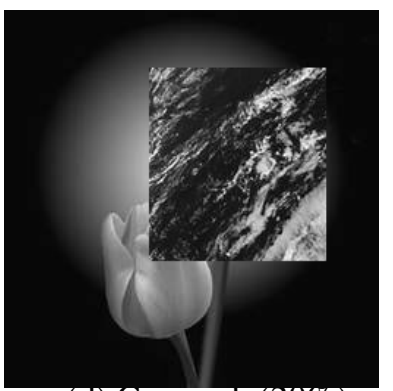

(d) Cropped $(20 \%)$

Fig. 8. Various attacks on watermarked image.

Table 1

Comparison of PSNR values for various attacks

\begin{tabular}{|c|c|c|c|}
\hline \multirow{2}{*}{$\begin{array}{c}\text { S1. } \\
\text { No. }\end{array}$} & \multicolumn{3}{|c|}{ PSNR Values in dB } \\
\cline { 2 - 4 } & Images & $\begin{array}{c}\mathrm{db} 2 \\
\text { filter }\end{array}$ & $\begin{array}{c}\text { Parametrized } \\
\text { filter }\end{array}$ \\
\hline 1 & Watermarked image & 51.87 & 56.45 \\
\cline { 2 - 4 } 2 & $\begin{array}{c}\text { Salt \& Pepper noise } \\
\text { added }\end{array}$ & 20.01 & 23.43 \\
\cline { 2 - 4 } 3 & Gaussian noise added & 18.02 & 20.25 \\
\cline { 2 - 4 } 4 & Median filtering & 21.06 & 23.36 \\
\cline { 2 - 4 } 5 & Cropping & 25.41 & 27.56 \\
\cline { 2 - 4 } & & &
\end{tabular}


Signal \& Image Processing : An International Journal (SIPIJ) Vol.3, No.1, February 2012

Table 2

Comparison of Similarity Measure values for various attacks

\begin{tabular}{|c|c|l|c|}
\hline \multirow{2}{*}{$\begin{array}{c}\text { Sl. } \\
\text { No. }\end{array}$} & Images & $\begin{array}{l}\text { db3 } \\
\text { filter }\end{array}$ & $\begin{array}{c}\text { Parametrized } \\
\text { filter }\end{array}$ \\
\hline 1 & Watermarked image & 0.9236 & 0.9334 \\
\cline { 2 - 4 } 2 & $\begin{array}{c}\text { Salt \& Pepper noise } \\
\text { added }\end{array}$ & 0.8521 & 0.8712 \\
\cline { 2 - 4 } 3 & Gaussian noise added & 0.8322 & 0.8395 \\
\cline { 2 - 4 } 4 & Median filtering & 0.7941 & 0.8122 \\
\hline 5 & Cropping & 0.8134 & 0.8225 \\
\cline { 2 - 4 } & \multicolumn{2}{|c}{} \\
\cline { 2 - 4 } & \multicolumn{2}{|c|}{}
\end{tabular}

\section{Conclusions}

A robust watermarking using filter parametrized RDWT and extraction using ICA is presented in this paper. The results obtained are compared with existing Daubechies filter to evaluate the performance of the proposed scheme. From the results, it is inferred that imperceptibility and robustness of the proposed scheme is improved interms of high PSNR values when compared to he conventional existing filter scheme. The proposed algorithm is adaptive by implementing $\mathrm{NVF}$, in which the watermark in texture and edges are stronger in flat areas. The advantage of using FastICA algorithm for watermark detection does not need information about location and strength parameters. The novelty of this ICA detection is that it extracts the watermark directly from spatial domain and not requires the transformation in the detection process. The proposed scheme can also be applied to biomedical watermarking and forensic images.

\section{REFERENCES}

[1] Wie-Bin Lee and Tung-Her Chen, (2002) "A public verifiable copy protection technique for still images",Journal of Systems and Software, Elsevier, Science Direct, vol. 62, pp. 195-204.

[2] Nguyen T.V. and Chandra patra J., (2007) "A simple ICA-based digital image watermarking scheme”, Science Direct Journal on Digital Signal Processing, vol.18, pp. 762-776.

[3] Wang Y., Dohert J.F.,Van Dyck E, (2002) “ A wavelet-based watermarking algorithm for ownership verification of digital images', IEEE Transactions on Image Processing, vol. 11, no.2, pp. 117-126.

[4] I.J.Cox, M.L.Miller, J.A. Bloom, J. Fridrich, and T. Kalkar, Digital watermarking and steganography, Second Edition, Morgan Kaufmann Publishers.

[5] T. D. Hien, Z. Nakao, and Y.W. Chen, (2006) "Robust multi-logo watermarking by RDWT and ICA,” Elsevier Journal on Signal Processing, vol. 86, pp. 2981-2993.

[6] S. Cui, and Y. Wang, (2006) "Redundant wavelet transform in video signal processing," in Proc. Int. Conf. Image Processing, Computer Vision and Pattern Recognition.

[7] D. Pollen, (1989) "Parametrization of compactly supported wavelets," Technical report, Aware Inc., USA.

[8] S. Voloshynovskiy, A. Herrigel, N. Baumgaertner, and T. Pun, (2000) "A stochastic approach to Content adaptive digital image watermarking", Lecture notes in Computer Science, vol. 1768, pp. 212-236, Springer, Berlin.

[9] A. Hyvarinen, J. Karhunen, and E. Oja, Independent component analysis, First Edition, John Wiley \& Sons, Inc., New York. 


\section{Authors}

P.MANGAIYARKARASI received her B.E., Electronics and Instrumentation, from Annamalai University in 1999 and M.E., Process control and Instrumentation from Annamalai University in 2001. Currently she is doing Ph.D. in the area of Digital Image Watermarking. At present she is working as Assistant Professor in the Dept. of Instrumentation Engg., Annamalai University. Her research interests include Digital Signal Processing, Digital Image Processing and Digital Watermarking.

S.ARULSELVI received her B.E., Instrumentation and Control from Govt. College of Technology, Coimbatore in 1988, M.E., Control and Instrumentation from Anna University in 1998 and Ph.D. in Power Electronics from Anna University in 2007. Currently she is working as Associate Professor in the Dept. of Instrumentation Engg., Annamalai University. Her area of interests includes Digital Watermarking, Image Processing, Power Electronics and Control etc.
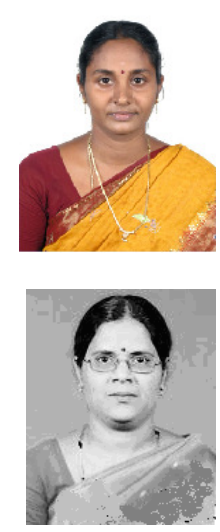